\title{
Evaluación del aprendizaje en educación física: relación entre las prácticas evaluativas de los docentes y enfoques de aprendizaje ${ }^{1}$
}

\author{
Marco Vinicio Gutiérrez ${ }^{2}$ \\ Universidad Manuela Beltrán, Universidad Libre ${ }^{3}$ \\ marcoviniciog@gmail.com \\ Luis Camilo Suárez Hernández ${ }^{4}$ \\ Secretaria de Educación (SED), Bogotá, Colombia ${ }^{5}$ \\ Kmilo.sh@gmail.com \\ Luis Ignacio Martínez Duarte ${ }^{6}$ \\ Secretaria de Educación (SED), Bogotá, Colombia ${ }^{7}$ \\ nachomartinez67@gmail.com
}

2 Doctor Honoris Causa. Filosofía de la educación. Consejo Ibero Americano (2010)

3 Docente investigador

4 Maestría en Ciencia y tecnología del deporte y la actividad física. Universidad Manuela Beltrán.

5 Docente

6 Maestría en ciencias y tecnología del deporte y la actividad física. Universidad Manuela Beltrán.

7 Docente 


\title{
Evaluación del aprendizaje en educación física: relación entre las prácticas evaluativas de los docentes y enfoques de aprendizaje
}

\section{Resumen}

El presente artículo muestra el resultado de una investigación que indagó sobre las prácticas evaluativas de los docentes de educación física, de los colegios públicos de la localidad de Suba en la ciudad de Bogotá, y la relación de estas prácticas con los enfoques superficial y profundo de aprendizaje. Está organizado en dos apartados, el primero presenta una caracterización de las prácticas evaluativas, y el segundo establece la relación de estas prácticas, con los enfoques superficial y profundo de aprendizaje. Este artículo deriva de una investigación con enfoque metodológico mixto, la muestra estuvo conformada por 68 docentes, como instrumento de recolección de información se aplicó una encuesta y su análisis se realizó utilizando el software estadístico R. Los resultados muestran el objeto, las finalidades, los procedimientos y los modos en que los docentes desarrollan su práctica evaluativa en educación física, y evidencian una tendencia hacia la promoción de aprendizaje profundo.

Palabras clave: Educación física, evaluación, prácticas evaluativas, aprendizaje superficial y aprendizaje profundo.

\section{Learning Assessment in physical education: the relationship between assessment practices of teachers and learning approaches}

\begin{abstract}
This article shows the result of an investigation that studied teachers' assessment practices in Physical Education in public schools from Suba in Bogota and the relationship between these practices with both superfluous and superficial learning approaches. It is organized into two sections; the first presents a classification of the evaluation practices, and the second establishes the relationship between these practices with the superficial and profound learning approaches. This article nourishes itself from a mixed-method research approach wherein the sample consisted of 68 teachers from whom data was collected using a survey. This data was then analyzed using the statistical software R. The results show the object, the purpose, procedures and ways in which teachers develop their assessment practice in physical education, and as well show a trend towards promoting meaningful and profound learning.
\end{abstract}

Keywords: physical education, evaluation, assessment practices, surface-to learning deep learning.

\section{Avaliação da aprendizagem na educação física: relação entre as práticas de avaliação dos docen- tes e as abordagens da aprendizagem}

\section{Resumo}

Este artigo apresenta o resultado de uma pesquisa sobre as práticas de avaliação dos docentes de Educação física, das escolas públicas da localidade de Suba, na cidade de Bogotá, e a relação dessas práticas com as abordagens superficial e profunda da aprendizagem. Ele está organizado em duas seções: a primeira apresenta uma caracterização das práticas de avaliação, e a segunda estabelece a relação dessas práticas com as abordagens superficial e profunda da aprendizagem. Este artigo é resultado de uma pesquisa com uma abordagem metodológica mista. A amostra esteve formada por 68 docentes. Como ferramenta de colheita da informação, foi realizada uma sondagem de opinião e, para a análise, foi usado o software estadístico R. Os resultados exibem o objeto, as finalidades, os procedimentos e os modos em que os docentes desenvolveram sua prática de avaliação na Educação Física, e evidenciam uma tendência à promoção da aprendizagem profunda.

Palavras chave: educação física, avaliação, práticas de avaliação, aprendizagem superficial-aprendizagem profunda. 


\section{Introducción}

La evaluación ha alcanzado en la última década gran importancia al interior de la comunidad educativa, es abordada por las diversas áreas del conocimiento, dentro de ellas la Educación Física (en adelante EF), que forman parte de las áreas fundamentales del currículo según lo establece la ley 115 (ley general de educación 1994) en Colombia. Incorporándola como componente esencial de procesos de formación y salud en el sistema educativo, desarrolla sus métodos a partir del énfasis en el cuerpo humano y su movimiento.

En Colombia, según el artículo 10 de la ley 181 de 1995 se entiende la EF como "[...] la disciplina científica cuyo objeto de estudio es la expresión corporal y la incidencia del movimiento en el desarrollo integral y en el mejoramiento de la salud y la calidad de vida de los individuos [...]" para Morales (2014) los lineamientos de la EF están dados desde el Ministerio de Educación Nacional con dos documentos, Los Lineamientos Curriculares (2000) y el documento número 15 (2010). Además, en Bogotá desde la Secretaria de Educación Distrital, El Currículo para la excelencia académica y formación Integral (2015). Estos documentos orientan el trabajo al interior del aula para el desarrollo de la EF y en los procesos de evaluación de los aprendizajes.

Específicamente en el ámbito evaluativo, el referente adoptado en este estudio retoma a Blázquez (2003), para quien la evaluación es "[...] un proceso dinámico, continuo y sistemático enfocado hacia los cambios de la conducta del alumno, mediante el cual verificamos los logros adquiridos en función de los objetivos propuestos $[\ldots . .]^{\prime \prime}$ (p.15). En el mismo sentido, el autor plantea cuatro componentes esenciales de la evaluación: el objeto de la evaluación que responde a la pregunta ¿Qué se evalúa? La finalidad de la evaluación que responde al ¿para qué se evalúa? Los procedimientos e instrumentos que responden al ¿cómo evaluar? Y los modos de evaluación, que se relacionan con la función, el momento, y la participación del alumno.

En el contexto educativo se realizan diferentes actividades, que hacen parte del componente didáctico: se evalúa, se califica y se miden aprendizajes, para entender el concepto de evaluación es necesario identificar las diferencias entre estos tres aspectos, al respecto Castillo y Cabrerizo (2003), dicen: "El concepto de evaluación es el más amplio de los tres, y engloba, por lo tanto, a los otros dos, pero no se identifica con ellos" (pp.14). La acción evaluadora tiende a confundirse con la medición y la calificación, si bien la medición es la tarea encaminada a recoger y registrar información de tipo cuantitativo, como indicador en una escala numérica o gradual de algún comportamiento o hecho que se pretenda medir sobre el aprendizaje, y la 
calificación como el símbolo numérico o letra que representa el grado en la escala de referencia de los aprendizajes pretendidos, la evaluación abarca muchos más aspectos.

\section{Perspectivas teóricas en torno al concepto de empatía}

El concepto de la empatía ha sido explicado por diferentes autores como la capacidad del individuo para "colocarse en el lugar del otro" de tal manera que le permita identificar sus sentimientos y emociones, hasta el punto de lograr experimentar lo mismo que éstos viven y experimentan. Desde los estudios realizados por (Lipps, 1903; Feshbach, 1983; Eisenberg y Strayer, 1992; Salmivalli, 1996; Chaux, 2004; Chaux, 2012;Gutiérrez, Escartí y Pascual, 2011) hasta los postulados más recientes propuestos por Deceaty (2014), la empatía ha sido considerada un aspecto fundamental para el desarrollo psicológico y emocional de los individuos, por cuanto es un factor que debe ser tenido en cuenta si se pretende propiciar ambientes de interacción social que ofrezcan a los individuos en formación entornos de armonía y sana convivencia. Para el presente trabajo, se retoma la conceptualización de empatía ofrecida por Gutiérrez et al. (2011, p.1) quienes la definen como "una respuesta afectiva de comprensión sobre el estado emocional de otros, que induce a sentir el estado en que se encuentra el otro". Al respecto, autores que han profundizado en su estudio, entre los que se encuentran Bandura y Hoffman citados por Gutiérrez et al. (2011) mencionan otro elemento que está involucrado en los procesos empáticos, relacionado con el componente cognitivo que implica un nivel de comprensión de esos estados afectivos de las otras personas, lo que ha llevado a considerar la empatía como un proceso multifuncional que involucra factores de índole emocional, cognitivo y social (Garaigordobil y García, 2006; Mestre et. al. 2002; González-Blasco, et al., 2013)

En el ámbito educativo, la evaluación, según Carreño (2010), recae sobre diferentes aspectos y actores: el sistema educativo, los estudiantes, los docentes, los procedimientos de enseñanza y aprendizaje, los directivos y las instalaciones, entre otros. Sin restarle importancia a estos componentes del contexto educativo, la aproximación teórica del presente artículo se centra en la evaluación del aprendizaje. La evaluación está centrada en el profesor, es él quien la planea y ejecuta, por lo tanto, la valoración de las realizaciones de los alumnos está determinada por las concepciones, tanto implícitas como explicitas que el docente tenga sobre los procesos de enseñar y aprender.

En relación directa con la evaluación está el aprendizaje, la forma en que aprenden los estudiantes nos aproxima a los enfoques de aprendizaje, a la manera en que los estudiantes abordan las situaciones propias del aprendizaje de una disciplina, en este 
caso la EF. Al respecto, algunos estudios hablan de enfoques superficial y profundo (Marton \& Saljo, 1976; Entwistle, 1981; Biggs, 2006; Morales, 1998). Según Biggs 2006, "[...] los enfoques superficial y profundo describen las dos formas que tienen los estudiantes de relacionarse con un ambiente de enseñanza y aprendizaje [...]" (pp.36). En el aprendizaje superficial, el estudiante tiene la intensión de liberarse de la tarea con el mínimo esfuerzo, y cumplir los mínimos requisitos exigidos, utilizando actividades de bajo nivel cognitivo. El aprendizaje profundo se genera por una motivación intrínseca, al abordar la tarea de manera adecuada y significativa, utilizando actividades apropiadas para conseguirla. Un apropiado procedimiento de enseñanza alinea los objetivos o propósitos de enseñanza con las actividades de aprendizaje, y estas con la evaluación, de manera que todos los aspectos promuevan el aprendizaje de los estudiantes. Para Fermín (2002), los procedimientos de evaluación del aprendizaje producen un efecto sobre las estrategias que adoptan los estudiantes en el aprendizaje mediante sus actividades de estudio.

El qué y el cómo aprenden los estudiantes tiene una relación con la forma en que serán evaluados. La evaluación debe propiciar un mensaje adecuado para que los estudiantes entiendan la evaluación como parte de su proceso de aprendizaje. Diversos estudios destacan la influencia de los sistemas de evaluación en los procesos de aprendizaje, y como efecto, en la calidad de los resultados alcanzados por los estudiantes. (Fermín, 2002; Soto, García \& Gonzales, 2012; Alcántara, 2013).

Los estudios sobre las prácticas evaluativas de los docentes de educación física son escasos en Colombia, sobresale la investigación a nivel nacional de Camacho (2011), Caracterización pedagógica en el área de educación física en básica secundaria del departamento del Huila. Estudio elaborado en 208 instituciones, los resultados indican la diferencia de conceptos de evaluación, la inclusión de la dimensión cognitiva, socio afectivo, motriz y psicomotriz de la evaluación. Otra contribución es la de Posada (2010), que hace una comparación de estudios preliminares y un contraste de las estrategias de evaluación usadas por los docentes en la ciudad de Manizales y el Municipio de Villa María, y su relación con las Políticas públicas de Colombia, este estudio fue realizado en 52 instituciones. Los resultados obtenidos fueron: los docentes conocen las normas vigentes en evaluación, pero: no son abordadas en su quehacer educativo; se evidencia la poca participación en los criterios de evaluación de los estudiantes; como recomendación a estos resultados, se propone la evaluación de carácter formativo e integral, así como la necesidad de implantar la auto evaluación, y la meta evaluación.

Regionalmente, en Bogotá, son escasos los estudios que dan cuenta de las prácticas evaluativas de los docentes de EF, en el estudio de Guio (2011), Las concepciones y prácticas evaluativas de los docentes de educación física de los colegios oficiales en la localidad de Usaquén, realizado a 16 docentes, y cuyos resultados dan cuenta de: la dificultad en la innovación de la evaluación debido a 
las prácticas tradicionales, la poca participación de los estudiantes en la proposición de criterios de evaluación, la observación como técnica más empleada por los docentes, el uso de los test como instrumento de diagnóstico y de verificación, la tendencia de privilegiar lo axiológico, la inclusión de lo actitudinal, el esfuerzo, el uniforme, y el aseo personal en la evaluación.

La relevancia del anterior estudio radica en conocer, la manera como los docentes de EF de los colegios públicos de la localidad de Suba en Bogotá, realizan los procesos de evaluación e inferir la relación que tienen estas prácticas con los enfoques de aprendizaje, generando un nuevo aporte que no se ha tenido en cuenta en investigaciones previas, esperando contribuir con las políticas públicas tendientes a mejorar la calidad educativa en Bogotá, y que den consistencia al área de EF.

\section{Método}

\section{Participantes}

Este estudio forma parte de un macro proyecto, que pretende incluir a los docentes de educación física de la ciudad de Bogotá. Para su realización se seleccionó una muestra de 68 docentes de una población total inicial de 83 docentes, de los cuales $36 \%$ son de género femenino y $63 \%$ masculino, pertenecientes a la Secretaria de Educación de Bogotá, y que laboran en colegios públicos en la localidad de Suba, en el área de EF en el nivel de básica secundaria y media, la edad de los docentes varía entre 24 y 59 años.

\section{Diseño y objetivos}

Este es un estudio con enfoque mixto, desde lo cuantitativo de tipo descriptivo transversal y cualitativo.

Los objetivos para la investigación se clasifican en dos grupos. En primer lugar, el objetivo relacionado con la caracterización de las prácticas evaluativas de los docentes de EF. En la que se incluye el objeto, las finalidades, los procedimientos y los modos que predominan en el desarrollo de la evaluación que ejecutan los docentes. Para la construcción de este objetivo, se tuvieron en cuenta el conjunto de respuestas de la encuesta en un análisis de correspondencia simple con el programa estadístico R, que nos permitió identificar las tendencias evaluativas de los docentes. El segundo objetivo se orienta hacia los enfoques de aprendizaje, en donde se analizó la relación que tienen las tendencias de las prácticas de evaluación de los docentes de EF, con los referentes teóricos y de investigación que dan cuenta sobre enfoques de aprendizaje superficial y profundo, que son promovidos con las prácticas de evaluación. 


\section{Técnicas e instrumentos de evaluación}

Para la recolección de los datos se empleó una encuesta, la cual se efectuó con preguntas abiertas y cerradas, de carácter autoadministrado. El instrumento diseñado se sometió a evaluación de tres expertos que hicieron recomendaciones puntuales, las cuales se atendieron para la realización de la prueba piloto. Finalmente, la encuesta se aplicó a la muestra referida. Para el análisis de resultados se hizo un Análisis de Contenido Sistemático ACS con el software R, que consistió en resumir los datos en un número reducido de dimensiones, con la menor pérdida de información posible. El instrumento aplicado consta de cuatro categorías: la primera se refiere al objeto de la evaluación en EF en la que se agrupan 15 preguntas, el segundo grupo responde a la finalidad agrupada en 8 preguntas, la tercera sobre procedimientos de evaluación 20 preguntas y finalmente los modos a través de 7 preguntas.

\section{Resultados}

Gráfico 1. Datos generales de la población.

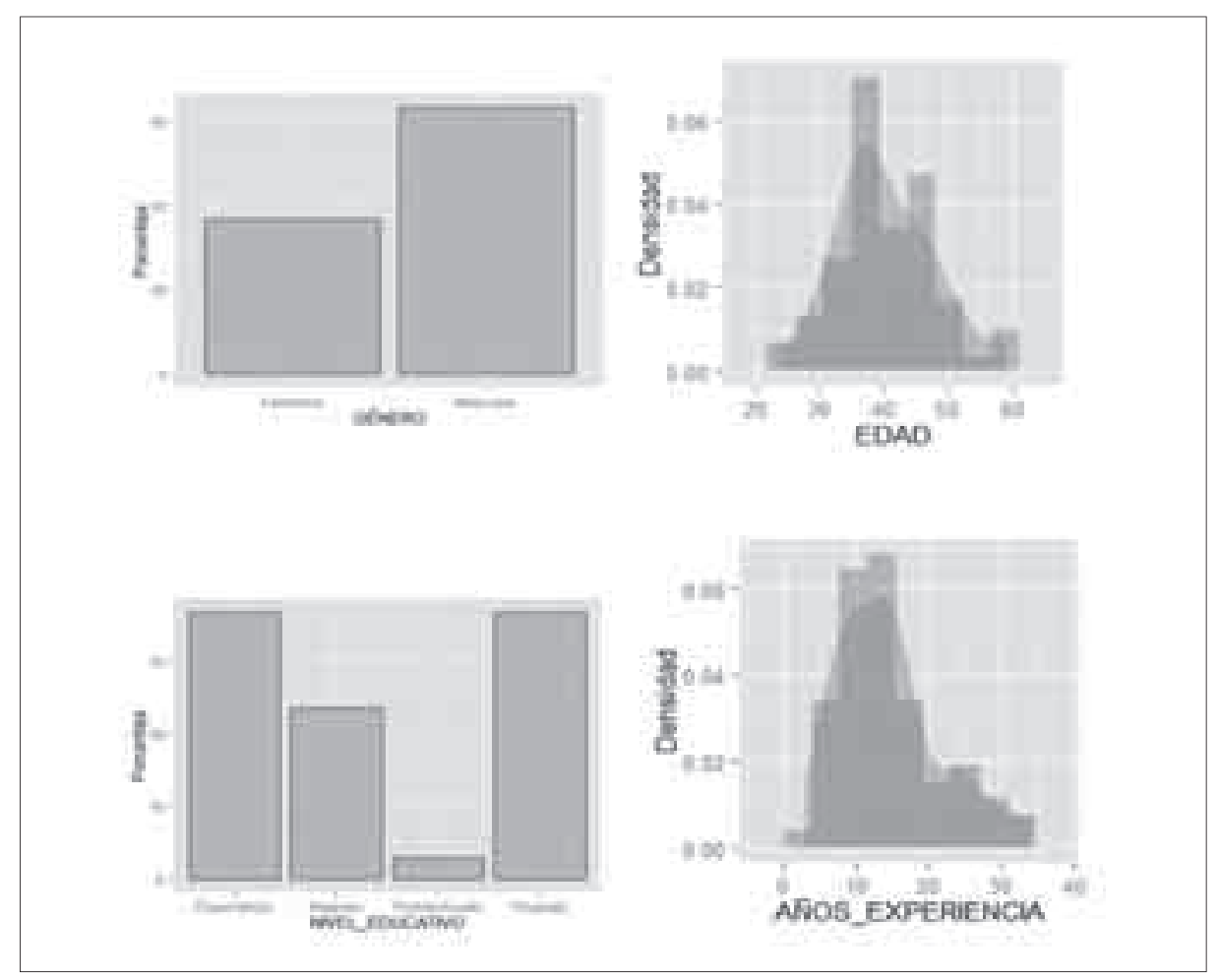


Tabla 1. Cuestionario: categoría uno, objeto de evaluación.

\section{Indique con qué frecuencia usted evalúa los estudiantes en los siguientes aspectos:}

A1: Estrategia deportiva

A2: Cumplimiento de las reglas establecidas en el grupo

A3: Composición corporal (porcentaje graso, peso, talla)

A4: Táctica deportiva

A5: Actitudes frente a los compañeros y el profesor

A6: Cualidades físicas, (fuerza, flexibilidad, etc.)

A7: Anatomía - fisiología

A8: Valores (responsabilidad, solidaridad, etc.)

A9: Habilidades motrices (equilibrio, ritmo, etc.)

A10: Conocimiento de aspectos propios de la disciplina (reglamentos, historia, etc.)

A11: Capacidad de liderazgo

A12: Habilidades técnicas (eficiencia técnica, dominio técnico)

A13: Conocimiento de hábitos de vida saludable

A14: Trabajo en equipo

A15: Expresión corporal (capacidad expresivo-comunicativa).

La primera categoría (tabla 1) en la encuesta se toma como un conjunto de preguntas de opción múltiple con única respuesta. Así, las 4 posibles opciones de respuesta en cada variable listadas se cruzan con las 15 preguntas dando paso a una matriz de respuestas como la que se muestra a continuación (tabla 2). 
Evaluación del aprendizaje en educación física: relación entre las prácticas evaluativas de los docentes y enfoques de aprendizaje | Marco Vinicio Gutiérrez | | Luis Camilo Suárez Hernández | | Luis Ignacio Martínez Duarte |

Tabla 2. Matriz de respuestas categoría uno, objeto de evaluación.

\begin{tabular}{|l|l|l|l|l|l|l|l|l|l|l|l|l|l|l|l|}
\hline & A1 & A2 & A3 & A4 & A5 & A6 & A7 & A8 & A9 & A10 & A11 & A12 & A13 & A14 & A15 \\
\hline MF. & 11 & 38 & 5 & 13 & 44 & 23 & 4 & 38 & 30 & 9 & 26 & 16 & 14 & 29 & 17 \\
\hline F. & 38 & 26 & 27 & 25 & 20 & 33 & 28 & 26 & 35 & 40 & 34 & 45 & 42 & 36 & 35 \\
\hline PF. & 14 & 4 & 29 & 24 & 3 & 9 & 26 & 3 & 3 & 18 & 8 & 6 & 11 & 3 & 16 \\
\hline NE. & 5 & 0 & 7 & 6 & 1 & 3 & 10 & 1 & 0 & 1 & 0 & 1 & 1 & 0 & 0 \\
\hline
\end{tabular}

Dónde, MF: Muy frecuente; F: Frecuente; PF: Poco frecuente; NE: No lo evalúo.

Gráfico 2. Promedios de respuestas categoría uno, objeto de evaluación.

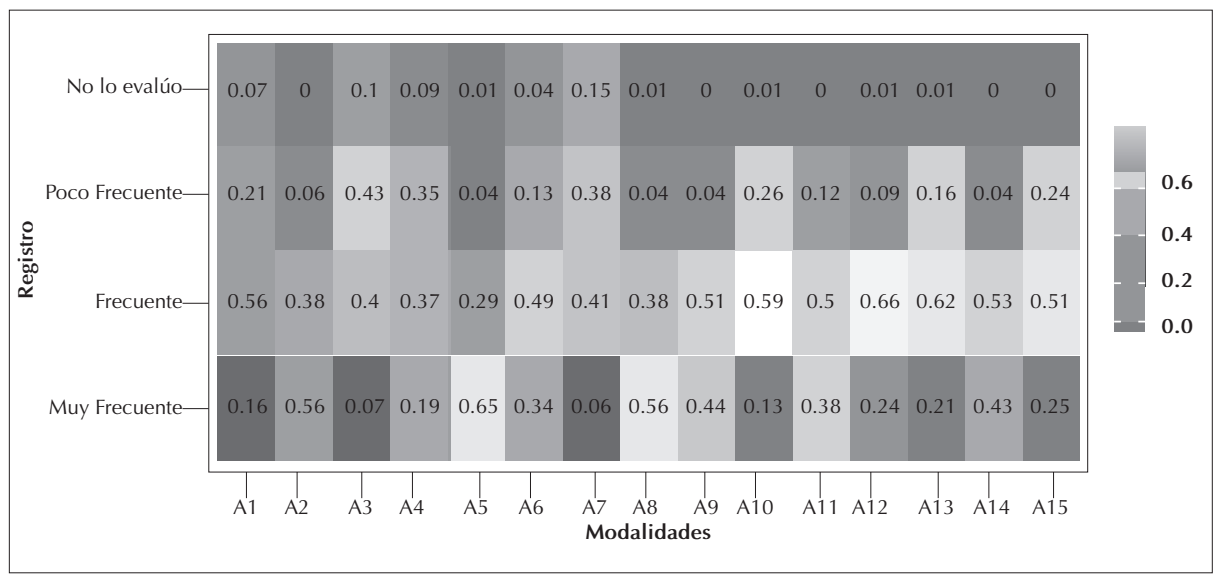

El gráfico 2 hace evidente que hay mayor tendencia a una evaluación frecuente seguida de una evaluación muy frecuente, es también bastante claro que hay una tendencia mínima a no evaluar. 
Gráfico 3. Análisis de correspondencia simple, diferenciada por colores, categoría uno.

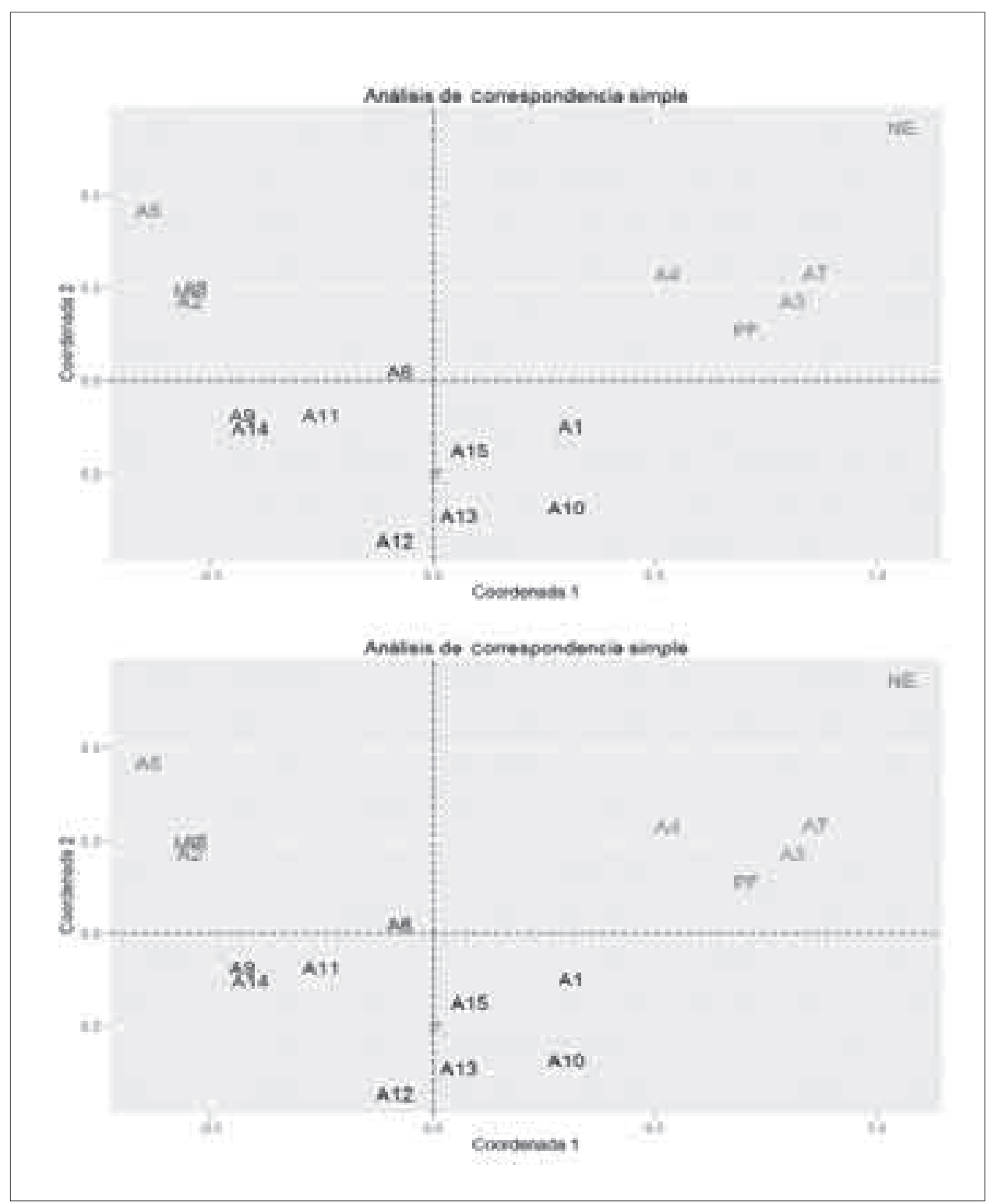

La primera dimensión del gráfico 3 está relacionada con la frecuencia de evaluación de los docentes, variando, de izquierda a derecha, de la mayor frecuencia a la no evaluación. Se puede apreciar una asociación muy fuerte entre las preguntas $\boldsymbol{A 2}, \boldsymbol{A} 5$ y $\boldsymbol{A} \boldsymbol{8}$ con la evaluación muy frecuente. De manera análoga se puede ver 
Evaluación del aprendizaje en educación física: relación entre las prácticas evaluativas de los docentes y enfoques de aprendizaje | Marco Vinicio Gutiérrez | | Luis Camilo Suárez Hernández | | Luis Ignacio Martínez Duarte |

que las preguntas $\boldsymbol{A} \mathbf{3}, \boldsymbol{A 4}$ y $\boldsymbol{A}$, presentan una asociación bastante fuerte con la evaluación poco frecuente.

Aunque el resto de preguntas parecen tener asociación con la evaluación frecuente, esto se puede malinterpretar debido a que cuando se reduce la cantidad de dimensiones, también se reduce la información que aportan los datos. En realidad, las preguntas que tienen mayor asociación con la no evaluación son $A 6, A 9, A 11$ y $A \mathbf{1 4}$, mientras que $A \mathbf{1}$, A10, A12, A13 y $A 15$ tienen mayor asociación con evaluación frecuente.

Tabla 3. Cuestionario, categoría dos sobre finalidades de la evaluación.

Indique la importancia que le otorga a las siguientes finalidades de la evaluación en educación física. Organice teniendo en cuenta que UNO (1) será la más importante y $\mathrm{OCHO}(8)$ la menos importante.

C1: Verificar que tanto ha aprendido el estudiante.

C2: Determinar el nivel de rendimiento en relación con determinados parámetros.

C3: Valorar que tanto ha servido su sistema de enseñanza.

C4: Detectar talentos deportivos.

C5: Motivar e incentivar al estudiante.

C6: Agrupar o clasificar los estudiantes de acuerdo con sus limitaciones o posibilidades.

C7: Asignar calificaciones.

C8: Obtener datos para investigar. 
Tabla 4. Matriz de respuestas, categoría dos, finalidades de la evaluación.

\begin{tabular}{|c|c|c|c|c|c|c|c|c|}
\hline$P$ & 01 & $\mathrm{O} 2$ & $\mathrm{O} 3$ & $\mathrm{O} 4$ & 05 & $\mathrm{O6}$ & 07 & 08 \\
\hline C1 & 12 & 8 & 22 & 13 & 5 & 4 & 1 & 3 \\
\hline $\mathrm{C} 2$ & 11 & 16 & 11 & 9 & 12 & 4 & 4 & 1 \\
\hline C3 & 10 & 14 & 8 & 12 & 11 & 4 & 7 & 2 \\
\hline C4 & 12 & 9 & 10 & 8 & 5 & 11 & 5 & 8 \\
\hline C5 & 29 & 17 & 4 & 8 & 6 & 1 & 2 & 1 \\
\hline C6 & 4 & 12 & 6 & 4 & 6 & 15 & 12 & 9 \\
\hline C7 & 4 & 5 & 4 & 6 & 9 & 13 & 9 & 18 \\
\hline C8 & 5 & 5 & 1 & 7 & 10 & 9 & 10 & 21 \\
\hline
\end{tabular}

Los niveles van desde $\boldsymbol{1}$ hasta $\boldsymbol{8}$ donde $\boldsymbol{1}$ es el más importante y $\boldsymbol{8}$ el menos importante.

Gráfico 4. Promedios de respuestas, categoría dos, finalidades de evaluación.

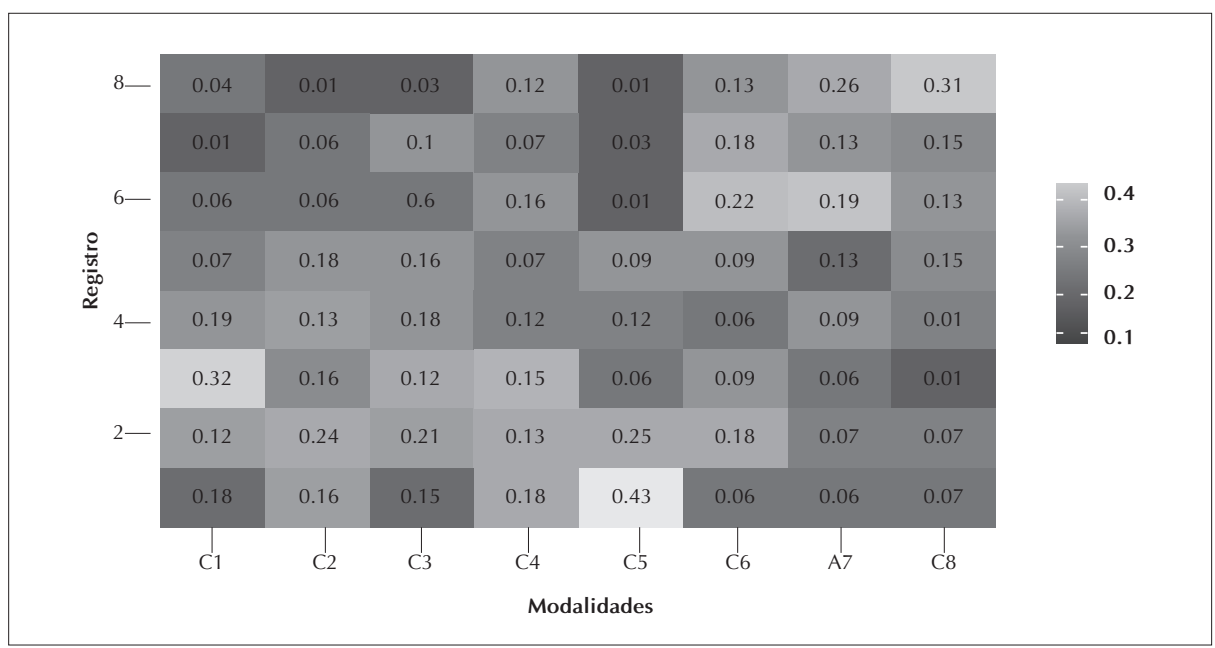


Evaluación del aprendizaje en educación física: relación entre las prácticas evaluativas de los docentes y enfoques de aprendizaje | Marco Vinicio Gutiérrez | | Luis Camilo Suárez Hernández | | Luis Ignacio Martínez Duarte |

En el gráfico 4 se hace evidente que hay mayor tendencia hacia el nivel 3 (importancia) seguido del nivel 2, nivel 1 y nivel 4 . Es claro que los niveles 5, 6, 7y $\boldsymbol{8}$ presentan una tendencia mínima.

Gráfica 5. Análisis de correspondencia simple, diferenciada por colores, categoría dos finalidades.

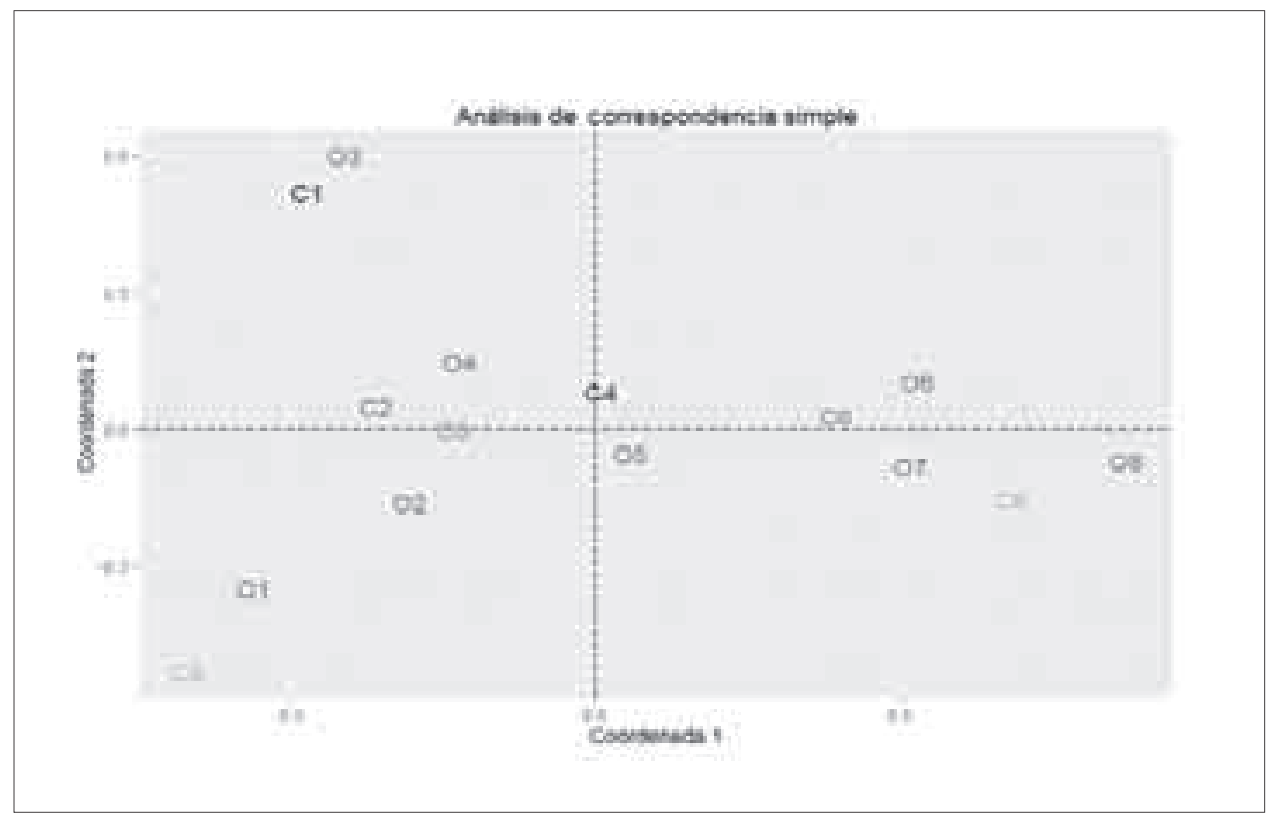

En la gráfica 5 se puede apreciar una asociación media entre la pregunta $C 1$ con el nivel de importancia 3. Las preguntas $C 2, C 3$, y $C 4$ muestran una asociación media con los niveles de importancia 2, 4 y 5 . De manera análoga se puede ver que las preguntas $\boldsymbol{C} \boldsymbol{6}, \boldsymbol{C 7}, \boldsymbol{C} \boldsymbol{8}$, presentan una asociación media con los niveles de importancia $\mathbf{6}, 7$ y $\boldsymbol{8}$. Finamente, podemos ver que existe una asociación media entre la pregunta $\boldsymbol{C 5}$, con el nivel de importancia $\mathbf{1 .}$

Tabla 5. Cuestionario, categoría tres, procedimientos de evaluación

¿Con qué frecuencia utiliza los siguientes procedimientos de evaluación en sus estudiantes a cargo?

E1: Comparación del desempeño del estudiante con listas de verificación de acciones o conductas.

E2: Comparación del desempeño del estudiante con escalas de clasificación o puntuación del rendimiento.

E3: Observación y registro de acciones o comportamientos. 
Tabla 5. Cuestionario, categoría tres, procedimientos de evaluación

E4: Cronometraje de la duración de un determinado comportamiento o acción.

E5: Comprobación periódica de la presencia de un comportamiento o acción.

E6: Pruebas escritas de conocimientos y datos.

E7: Pruebas escritas de verdadero falso.

E8: Pruebas escritas de selección múltiple.

E9: Pruebas escritas de apareamiento, por ejemplo dos columnas de términos en donde se deben unir con líneas los que sean completarías.

E10: Pruebas de identificación a través de videos, láminas, etc.

E11: Pruebas escritas con preguntas abiertas.

E12: Exámenes orales.

E13: Evaluación en grupo.

E14: Pruebas de ejecución de habilidades técnicas o tácticas.

E15: Seminarios, debates.

E16: Festivales, mini torneos.

E17: Exposiciones.

E18: Cumplimiento de tareas autónomas asignadas al estudiante.

E19: Evaluación entre compañeros de clase o coevaluación.

E20: Auto evaluación del estudiante. 
Evaluación del aprendizaje en educación física: relación entre las prácticas evaluativas de los docentes y enfoques de aprendizaje | Marco Vinicio Gutiérrez | | Luis Camilo Suárez Hernández | | Luis Ignacio Martínez Duarte |

Gráfico 6. Promedios de respuestas procedimientos de evaluación.

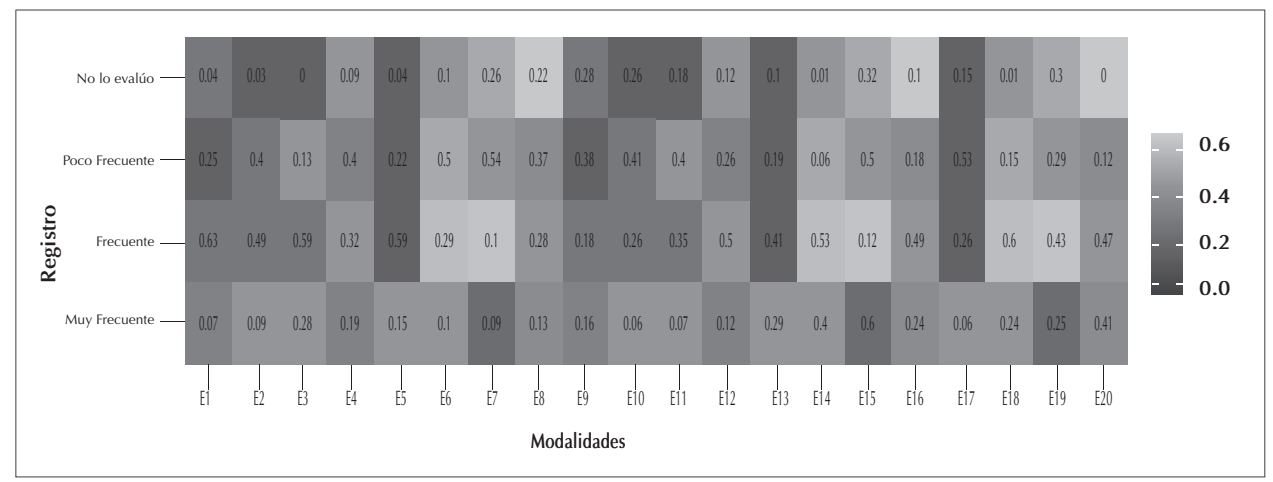

En el grafico 6 se hace evidente que hay mayor tendencia a una evaluación frecuente seguida de una evaluación poco frecuente, es también bastante claro que hay poca tendencia evaluar de manera muy frecuente o no evaluar.

Gráfico 7. Análisis de correspondencia simple, diferenciada por colores procedimientos evaluación

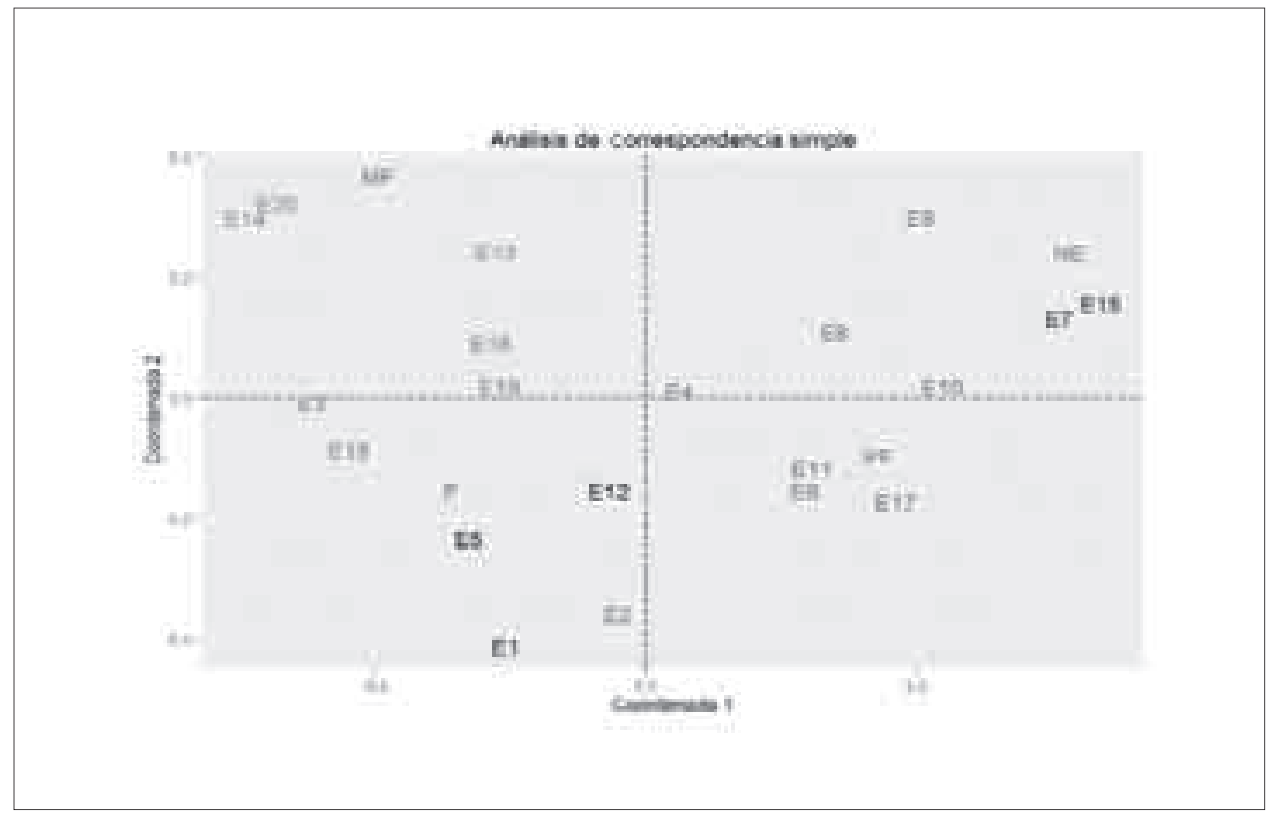

La primera dimensión del gráfico 7 está relacionada con la frecuencia de evaluación de los docentes, variando de izquierda a derecha, de la mayor frecuencia a la no evaluación. 
Se puede apreciar una asociación fuerte entre las preguntas E3, E13, E14, E16, E18, E19 y $\boldsymbol{E} 20$ con la evaluación muy frecuente. De manera análoga se puede ver que las preguntas $\boldsymbol{E 1}, \boldsymbol{E} 5$ y $\boldsymbol{E} \mathbf{1 2}$, presentan una asociación bastante fuerte con la evaluación frecuente, aunque realmente $\boldsymbol{E} \boldsymbol{9}$, como se verá más adelante, tiene más asociación con evaluación poco frecuente. En el caso de la no evaluación, la tendencia es a estar asociado con las preguntas E7, E9 y E15, mientras que, para la poca frecuencia de la evaluación la asociación es con las preguntas E2, E4, E6, E8, E10, E11 y E17. Una vez se han identificado los grupos, la anterior afirmación sobre la asociación entre la pregunta $\boldsymbol{E 9}$ y la poca frecuencia de evaluación se hace evidente, además, se pueden diferenciar mediante colores en el gráfico de correspondencias simple.

Tabla 6. Tabla 6. Categoría cuatro, modos de evaluación.

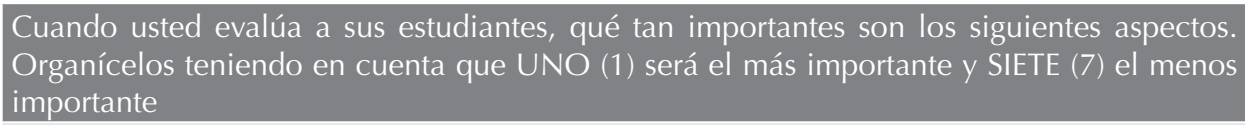

F1: Proporcionar al estudiante diversas oportunidades para demostrar su aprendizaje.

F2: Establecer criterios claros para que el estudiante los conozca y así, se puedan obtener las calificaciones.

F3: Tener en cuenta la normatividad institucional.

F4: Permitir la participación del estudiante en el proceso de evaluación (coevaluación y autoevaluación).

F5: Determinar los conocimientos previos de los estudiantes para diseñar la enseñanza y la evaluación.

F6: Clasificar los estudiantes según los desempeños altos - bajos - medios.

F7: Hacer de la evaluación una oportunidad de aprendizaje.

Tabla 7. Matriz de respuestas categoría cuatro, modos de evaluación.

\begin{tabular}{|c|c|c|c|c|c|c|c|}
\hline P & O1 & O2 & O3 & O4 & O5 & O6 & O7 \\
\hline F1 & 12 & 8 & 22 & 13 & 5 & 4 & 1 \\
\hline F2 & 11 & 16 & 11 & 9 & 12 & 4 & 4 \\
\hline F3 & 10 & 14 & 8 & 12 & 11 & 4 & 7 \\
\hline F4 & 12 & 9 & 10 & 8 & 5 & 11 & 5 \\
\hline F5 & 29 & 17 & 4 & 8 & 6 & 1 & 2 \\
\hline F6 & 4 & 12 & 6 & 4 & 6 & 15 & 12 \\
\hline F7 & 4 & 5 & 4 & 6 & 9 & 13 & 9 \\
\hline
\end{tabular}

Los niveles van desde $\mathbf{1}$ hasta 7 donde 1 es el más importante y 7 el menos importante 
Evaluación del aprendizaje en educación física: relación entre las prácticas evaluativas de los docentes y enfoques de aprendizaje | Marco Vinicio Gutiérrez | | Luis Camilo Suárez Hernández | | Luis Ignacio Martínez Duarte |

Gráfico 8. Promedios de respuestas categoría cuatro modos evaluación.

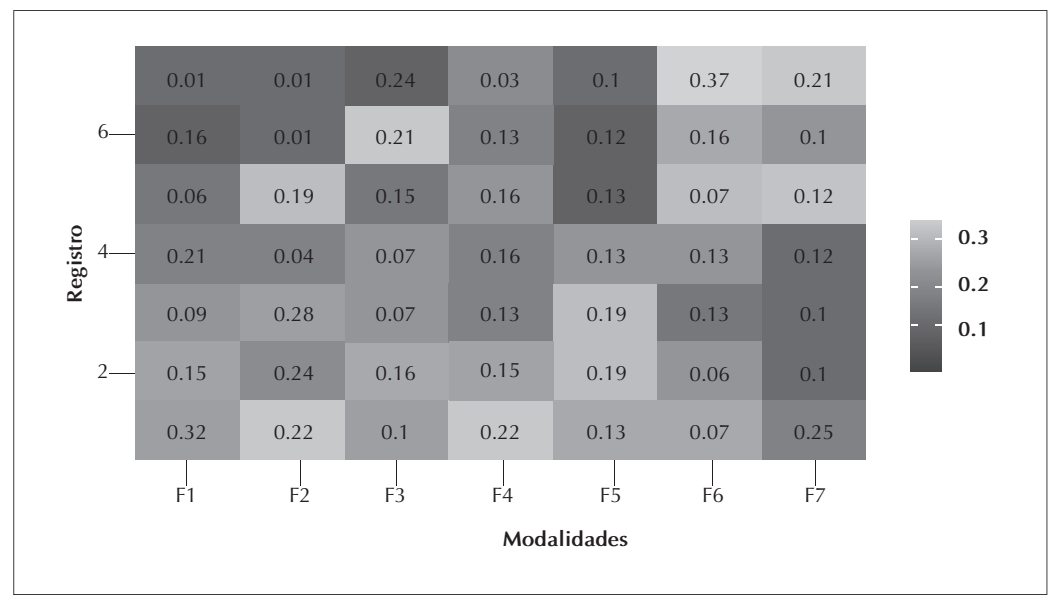

En el gráfico 8 se hace evidente que hay una mayor tendencia hacia el nivel 1 (importancia) seguido del nivel 2, nivel 3, nivel 4 y nivel 6. Los demás niveles presentan una tendencia mínima.

Gráfico 9. Análisis de correspondencia simple, categoría cuatro, modos evaluación.

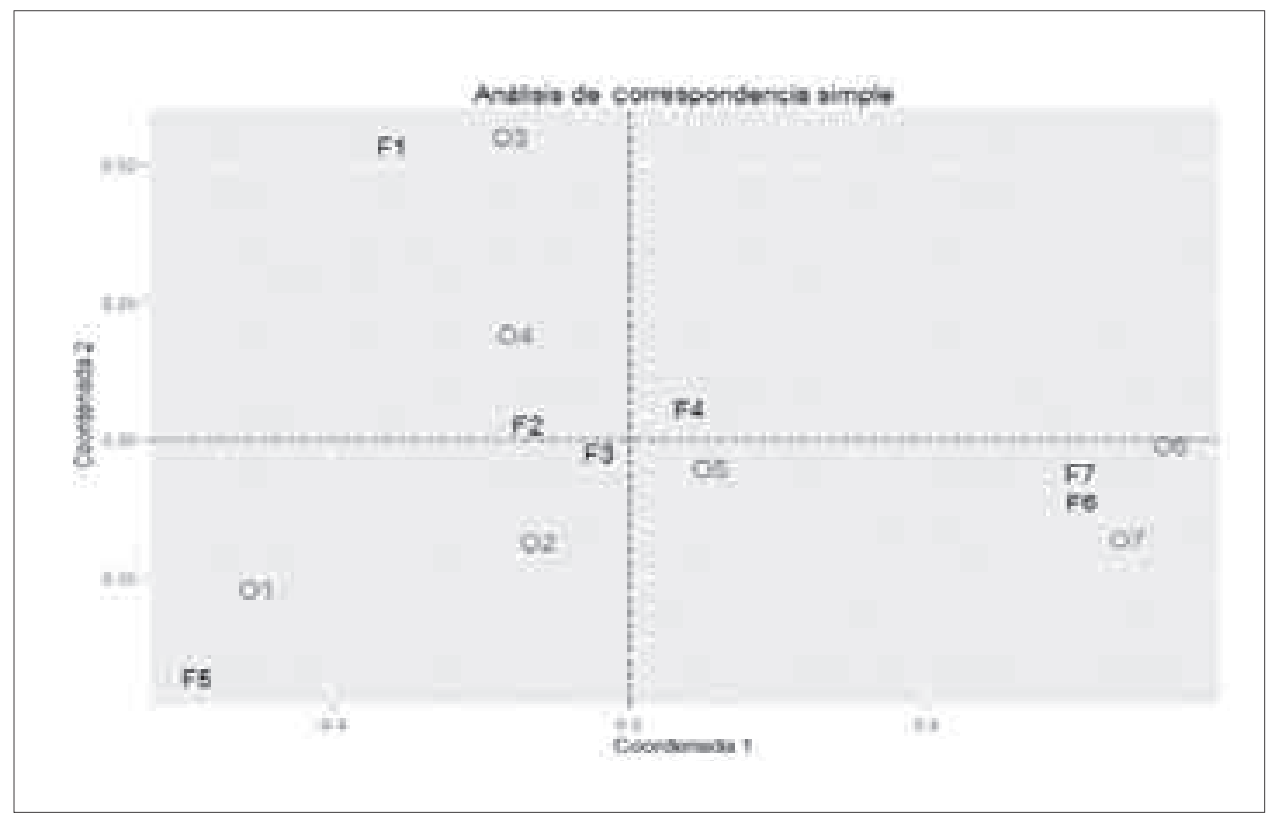


En el gráfico 9 se puede apreciar una asociación media, entre la pregunta $\boldsymbol{F 1}$ con el nivel de importancia 3. Las preguntas $F 2$, F3, y F4 muestran una asociación media con los niveles de importancia 2, 4 y 5. De manera análoga, se puede ver que las preguntas $\boldsymbol{F} \mathbf{6}$, y $\boldsymbol{F}$, presentan una asociación media con los niveles de importancia $\mathbf{6}$, y 7 . Finalmente, podemos ver que existe una asociación media entre la pregunta $F 5$ con el nivel de importancia 1.

\section{Discusión}

Al caracterizar las prácticas evaluativas de los docentes de EF de la localidad de Suba, se determinó: el objeto, las finalidades, los procedimientos y los modos que predominan en el desarrollo de la evaluación. Las caracteristicas obtenidas es posible relacionarlas con los referentes teóricos que tienen acercamientos respecto al aprendizaje superficial y profundo, y se establece una tendencia hacia un enfoque de aprendizaje, establecido por el predominio de determinadas prácticas evaluativas como se describe a continuación:

El objeto de la evaluación responde a ¿qué evalúan los docentes? Se observa que el ámbito social afectivo presenta la mayor tendencia, es decir, que la prioridad expresada por los docentes de EF en la localidad de Suba, responde al componente axiológico, los aspectos actitudinales, de valores y normas adquieren una relevancia, que se ve reflejada en la intención de evaluar este componente con mayor frecuencia, y que responde a las propuestas generadas desde los documentos guías emitidos desde el Ministerio de Educación Nacional, y la Secretaria de Educación Distrital. En un segundo nivel de importancia se ubica el ámbito cognitivo y motor, los conocimientos propios de la disciplina que amplían el bagaje de información y cultura, tanto deportiva como de hábitos que inciden en estilos de vida saludables. Las habilidades y expresiones motrices básicas y específicas, son para los docentes de EF un componente que se evalúa con regular frecuencia. El ámbito deportivo es considerado por los docentes como el de menor importancia en sus procesos de evaluación, es decir, que el deporte no es una prioridad para los procesos de enseñanza y aprendizaje en la EF en los colegios públicos de la localidad de Suba.

En relación con el propósito de la evaluación, ¿para qué evaluar? Los docentes utilizan la evaluación con intenciones formativas, en la que se involucra a los estudiantes de manera intrínseca en su aprendizaje y no exclusivamente para medir cuantitativamente las realizaciones. Se hace evidente en los resultados del estudio, la mayor tendencia de los docentes de EF de la localidad de Suba, a utilizar la evaluación para motivar e incentivar, en oposición a una menor tendencia a otorgarle a la evaluación la finalidad de asignar calificaciones, clasificar o agrupar estudiantes por sus resultados. Otra finalidad a la que se le da una significativa importancia, es verificar que tanto se ha aprendido, determinar el rendimiento de los estudiantes con determinados parámetros y valorar la eficacia de sus sistemas de enseñanza. La detección de talentos deportivos como propósito de la evaluación presenta una baja tendencia. 
Los procedimientos de evaluación dan cuenta de cómo se evalúa, un sistema de enseñanza y aprendizaje debe articular de manera coherente, los objetivos, con las actividades de aprendizaje, y estas con una evaluación adecuada y con la intención de propiciar aprendizajes que perduren, promoviendo en los estudiantes formas intrínsecas de afrontar las actividades de estudio. Los procedimientos de evaluación generan diferentes formas de asimilar los aprendizajes, los resultados de la investigación evidencian que los docentes de EF de Suba, emplean con mayor frecuencia procedimientos de observación y registro de comportamientos, el cumplimiento de tareas autónomas, la autoevaluación y coevaluación, así como pruebas de ejecución de habilidades técnicas o tácticas. En oposición los procedimientos con baja frecuencia son: las pruebas escritas, y el cronometraje de determinadas acciones, los procedimientos que tienen una tendencia a no ser evaluados son las pruebas escritas de falso o verdadero, los debates y seminarios.

Se observa una definida tendencia a emplear procedimientos cualitativos de tipo subjetivo en la evaluación según Blázquez (2003) “[...] en educación física podemos hablar de dos grandes tipos de evaluación objetiva y subjetiva y de dos técnicas de medición cuantitativas y cualitativas. La evaluación subjetiva es aquella que depende prioritariamente del juicio del profesor y cualitativa se entiende aquella que se basa en una medida mental dependiendo del examinador [...]" (p.p. 59-60).

En cuanto a la categoría relacionada con el modo de evaluación, que responde a cuatro elementos: según el tiempo, según la normatividad, según el ámbito de aplicación pruebas internas y externas, y según la participación del estudiante. Los docentes de EF manifiestan una tendencia positiva a la evaluación continua formativa, de igual manera predomina la evaluación criterial sobre la normativa, y destacan la importancia de permitir la participación de los estudiantes en su propio proceso de evaluación. Los resultados de los modos en el estudio, infieren una tendencia de utilizar los que promueven la participación autónoma, que influye positivamente para que el estudiante asuma y se interese en sus propios procesos de aprendizaje.

En relación con el segundo objetivo, concerniente a las prácticas evaluativas y su relación con los enfoques de aprendizaje superficial y profundo, podemos afirmar que teniendo en cuenta: el objeto de la evaluación hacia una tendencia de lo formativo (ámbito afectivo social), el uso de procedimientos e instrumentos de evaluación hacia técnicas cualitativas de tipo subjetivo y estrategias meta cognitivas (autoevaluación y coevaluación), y tomando como referencia las investigaciones de los procesos de evaluación y su relación con el enfoque de aprendizaje planteado por Alcántara (2013), que afirma que la forma en que el profesorado plantea la evaluación de su alumnado afecta a los enfoques de aprendizaje superficial y profundo. En el mismo sentido Hernández, Martínez \& Rubio (2005), plantean que la calidad de los aprendizajes está directamente relacionada con el enfoque de aprendizaje adoptado por el estudiante y promovido por el docente. Unas estrategias evaluativas cuantitativas Ilevan a enfoques superficiales de aprendizaje, mientras que 
las estrategias formadoras y cualitativas pueden producir enfoques de aprendizaje profundo y de alto rendimiento (Bordas \& Cabrera, 2001).

Los resultados hacen evidente un propósito de formación desde la EF de las diferentes dimensiones de los estudiantes, a diferencia de lo que se pudiera deducir a priori, los docentes de EF, no enfatizan en el desarrollo de lo físico, las habilidades y destrezas de forma exclusiva. La fortaleza de la EF, se justifica al integrar las dimensiones del ser humano como una totalidad que se desarrolla desde el planteamiento de los objetivos, pasando por las actividades propias de aprendizaje y fundamentada en la evaluación pensada, planeada y ejecutada en un proceso continuo. La importancia de una evaluación que se integre durante todo el proceso didáctico como componente formativo, requiere estrategias de evaluación formativas, no se pueden argumentar procesos formativos que pretenden mejorar la calidad educativa, con prácticas evaluativas que no son adecuadas para este objetivo en la EF.

Pretender mejorar los resultados de los estudiantes debe pasar por reflexionar hacia unas prácticas de evaluación, que se integren intencionalmente en el proceso didáctico y que promuevan enfoques de aprendizaje profundo, transformando la manera en que los estudiantes se enfrenten a sus tareas de estudio, en aras de mejorar la calidad educativa, y que pueda ser extendido a todas las áreas establecidas en el plan de estudios. Promover una evaluación formativa en EF, que valore de manera integral el ámbito social afectivo, cognitivo y motor, y que involucre de manera intrínseca y reflexiva al estudiante, permitiéndole reconocer sus fortalezas y debilidades. Todo esto, teniendo claro los objetivos de aprendizaje pretendidos, para involucrarlo y motivarlo positivamente, en la manera como enfrenta y asume estrategias de estudio, que favorezcan una profundidad en la asimilación de los aprendizajes y en consecuencia, la calidad educativa pretendida. 


\section{Referencias}

Alcántara, N. (2013). Proceso de evaluación y su relación con el enfoque de aprendizaje, Recuperado de http://www.unitec.edu/innovare/?p=220

Biggs, J. (2006). Calidad del aprendizaje universitario. España: Narcea.

Blázquez, D. (2003). Evaluar en educación física. España: Inde publicaciones.

Camacho, H. (2011). Caracterización pedagógica del área de educación física en básica secundaria en el Departamento del Huila. Entornos. 24, 123-138.

Carreño, F. (2010). Enfoques y principios teóricos de la evaluación. Barcelona: Trillas.

Castillo, S. (2003). Evaluación Educativa y Promoción Escolar. Madrid: Prentice Hall Pearson Educación.

Ley 115 de 1994. Ley general de educación, Congreso de Colombia, Bogotá, Colombia, 8 de Febrero de 1994.

Fermín, N. (2002). La Evaluación del Aprendizaje y su influencia en el comportamiento estratégico del estudiante universitario. Contextos Educativos, (5) 56-63.

Gutiérrez, F. (2011). Concepción y prácticas evaluativas de los docentes de Educación Física en colegios distritales de la localidad de Usaquén. Tesis de maestría no publicada. Universidad de la Sabana, Facultad de Educación, Bogotá, Colombia.

Hernández, S. (2010). Metodología de la Investigación. México: McGraw-Hill.

República de Colombia. Ley 181 de 1995. Art. 10. Creación del Sistema Nacional del Deporte. Bogotá. Recuperado de http://www.alcaldiabogota.gov.co/sisjur/normas/Norma1. jsp? $\mathrm{i}=3424$

Ministerio de Educación Nacional. (2000). Serie de Lineamientos Curriculares Educación Física Recreación y Deporte. Recuperado de http://www.mineducacion.gov.co/1621/ articles-89869_archivo_pdf3.pdf

Ministerio de Educación Nacional. (2010). Orientaciones pedagógicas para la recreación y deporte. Recuperado de http://www.mineducacion.gov.co/1621/articles-209504_ archivo_pdf_orientaciones_edufisica.pdf

Morales, C. (2014). Una mirada a la Educación Física en Colombia. Recuperado de http:// www.efdeportes.com/efd190/la-educacion-fisica-en-colombia.htm

Morales, P. (1998). Evaluación y aprendizaje de calidad. Guatemala: Universidad Rafael Landivor.

Muggenburg M. (2007). Tipo de estudio en el enfoque de investigación cuantitativa. Éneo. $4(1), 35-38$.

Posada, W. (2010). La evaluación de la Educación Física y las políticas educativas en Colombia. avatares entre la mente, el papel y el contexto. El caso de Manizales y Villamaria (Caldas). Revista Iberoamericana de Educación. 53(1), 1-11. 
República de Colombia. Ministerio de Salud. Resolución 008430 de 1993. Por la cual se establecen las normas científicas, técnicas y administrativas para la investigación en salud. Bogotá: Recuperado de http://www.comisionseptimasenado.gov.co/salud/SALUD\%20 CUADRO\%20RESOLUCIONES\%201993\%20AL\%202008\%20-\%202.pdf

Secretaria de Educación del Distrito Alcaldía Mayor de Bogotá. (2014). Currículo para la excelencia académica y la formación integral orientaciones para el área de Educación Física. Recuperado de http://www.educacionbogota.edu.co/archivos/Temas\%20estrategicos/ Documentos/DOCUMENTO_DEPORTES_MARZO_21.pdf

Soto, J., García, M., Gonzales, S. (2012). Enfoques y estrategias de aprendizaje: un binomio para comprender el rendimiento en la educación secundaria. Revista de Investigación en Educación. (10), 95-108.

Recibido: 13 de mayo 2014

Aceptado: 12 de agosto 2014

Como citar:

Gutiérrez, M.V., Suárez, L.C. y Martínez, L.I. (2015) Evaluación del aprendizaje en educación física: relación entre las prácticas evaluativas de los docentes y enfoques de aprendizaje. Praxis Pedagógica, 16, 53-74. 